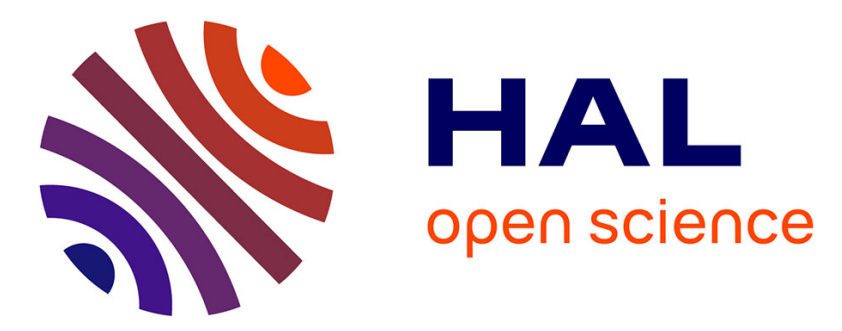

\title{
The Rule-based Conceptual Design of the Architecture of Serial Schonflies-motion Generators
}

Stéphane Caro, Waseem A. Khan, Damiano Pasini, Jorge Angeles

\section{To cite this version:}

Stéphane Caro, Waseem A. Khan, Damiano Pasini, Jorge Angeles. The Rule-based Conceptual Design of the Architecture of Serial Schonflies-motion Generators. Mechanism and Machine Theory, 2010, 45 (2), pp.251-260. 10.1016/j.mechmachtheory.2009.09.002 . hal-00464305

\section{HAL Id: hal-00464305 https://hal.science/hal-00464305}

Submitted on 16 Mar 2010

HAL is a multi-disciplinary open access archive for the deposit and dissemination of scientific research documents, whether they are published or not. The documents may come from teaching and research institutions in France or abroad, or from public or private research centers.
L'archive ouverte pluridisciplinaire HAL, est destinée au dépôt et à la diffusion de documents scientifiques de niveau recherche, publiés ou non, émanant des établissements d'enseignement et de recherche français ou étrangers, des laboratoires publics ou privés. 


\title{
The Rule-based Conceptual Design of the Architecture of Serial Schönflies-motion Generators
}

\author{
Stéphane Caro* \\ stephane.caro@irccyn.ec-nantes.fr \\ Waseem Ahmad Khan ${ }^{\dagger} \quad$ Damiano Pasini $^{\ddagger} \quad$ Jorge Angeles $^{\ddagger}$ \\ waseem@robotmaster.com damiano.pasini@mcgill.ca angeles@cim.mcgill.ca \\ * Institut de Recherche en Communications et Cybernétique de Nantes \\ UMR CNRS n 6597 \\ 1 rue de la Noë, 44321 Nantes, France \\ $\dagger$ Jabez Technologies Inc. \\ 5929 Rte Transcanadienne, suite 320, St. Laurent, QC, H4T 1 Z6 Canada \\ $\ddagger$ Department of Mechanical Engineering \& Centre for Intelligent Machines \\ McGill University \\ 817 Sherbrooke West, Montreal, QC, H3A 2K6 Canada
}

\begin{abstract}
The conceptual design of robotic architectures, the subject of this paper, pertains to the topology of the underlying kinematic chain. Schönflies Motion Generators (SMGs) are robots capable of a special class of motions - three independent translations and one rotation about an axis of fixed direction. In this paper, synthesis rules are proposed to obtain a complete minimum set of serial topologies capable of producing Schönflies motions. Only revolute $(\mathrm{R})$, prismatic $(\mathrm{P})$, helical $(\mathrm{H})$ and $\Pi$ joints are considered, as any multi-degree-of-freedom lower kinematic pair can be produced as a combination of $\mathrm{R}, \mathrm{P}, \mathrm{H}$ and $\Pi$ joints. Next, the concept-evaluation framework, proposed in an earlier work, is used to organize the topologies obtained in an ascending order of complexity, hence completing the conceptual design phase of the subject motion generators.
\end{abstract}


Keywords: Kinematic Synthesis; Conceptual Design; Schönflies Motion Generators; Complexity.

\section{Introduction}

The conceptual design of the architecture of a mechanical system aims at defining the topology of the associated kinematic chain. Schönflies-Motion Generators (SMGs) are robots capable of a special class of motions, those produced with serial robots termed SCARA (SelectiveCompliance Assembly Robot Arm) [1]: three independent translations and one rotation about an axis of fixed direction, similar to the motions undergone by the tray of a waiter. This set of displacements was first studied by the German mathematician-mineralogist Arthur Moritz Schönflies (1853-1928). For this reason, such motions are termed Schönflies. They are known to stem from a displacement subgroup of rigid-body motions, termed the Schönflies subgroup [2, 3, 田. The set of displacements of Schönflies motions is endowed with the algebraic properties of a group [5, 6].

A list of serial SMGs was produced by Lee and Hervé [4, 7, 8] based on the Lie-groupalgebraic properties of the displacement set. However, Lee and Hervé's list does not contain architectures with $\Pi$-joints. The latter are also called parallelogram joints because a $\Pi$-joint is a four-bar linkage with its opposite links of the same lengths. Although it might appear counterintuitive to regard the parallelogram linkages of interest as joints, the term is completely justified, as these joints (i) have appeared in many industrial robots, notably those with the Delta architecture [9] and the Quattro [10]; (ii) are well documented in the technical literature [11, 12, 13]; and (iii) have been studied systematically as valuable means in parallel-robot design [5].

In this paper, synthesis rules are proposed to obtain a complete minimum set of serial topologies capable of producing Schönflies motion. Only revolute $(\mathrm{R})$, prismatic $(\mathrm{P})$, helical $(\mathrm{H})$ and $\Pi$ joints are considered. In fact, any of the remaining lower kinematic pairs (LKPs) can be synthesized as a combination of R, P, H and $\Pi$ joints. Next, the concept-evaluation framework, proposed in an earlier work, is used to organize the topologies obtained in an ascending order of complexity, hence completing the conceptual design phase of the subject motion generators.

\section{Kinematic Bond, Kinematic Chain and Kinematic Pair}

It is known [3] that the set of rigid-body displacements $\mathcal{D}$ has the algebraic structure of a group. Moreover, $\mathcal{D}$ includes interesting and practical subgroups that find relevant applications in the design of production-automation and prosthetic devices. The combination of subgroups, 
in general, can take place via the standard set operations of union and intersection. The set defined as that comprising the elements of two displacement subgroups is not necessarily a subgroup, and hence, one cannot speak of the union of displacement subgroups. On the contrary, the intersection of two displacement subgroups is always a subgroup itself, and hence, the intersection of displacement subgroups is a valid group operation. Rather than the union of groups, what we have is the product of groups [14. Let $\mathcal{G}_{1}$ and $\mathcal{G}_{2}$ be two groups defined over the same binary operation $\star$; if $g_{1} \in \mathcal{G}_{1}$ and $g_{2} \in \mathcal{G}_{2}$, then the product of these two groups, represented by $\mathcal{G}_{1} \bullet \mathcal{G}_{2}$, is the set of elements of the form $g_{1} \star g_{2}$. Here, the order is important, as commutativity is not to be taken for granted in group theory, i.e., in general, $\mathcal{G}_{1} \bullet \mathcal{G}_{2} \neq \mathcal{G}_{2} \bullet \mathcal{G}_{1}$.

The intersection of the two foregoing groups, represented by the usual set-theoretic symbol $\cap$, i.e., $\mathcal{G}_{1} \cap \mathcal{G}_{2}$, is the group of elements $g$ belonging to both $\mathcal{G}_{1}$ and $\mathcal{G}_{2}$, and hence, the order is not important. Thus, $\mathcal{G}_{1} \cap \mathcal{G}_{2}=\mathcal{G}_{2} \cap \mathcal{G}_{1}$.

A total of 12 displacement subgroups of the group of rigid-body displacements was first listed by Hervé [15]. For quick reference, we reproduce Hervé's list below:

1. $\mathcal{R}(\mathcal{A})$, the subgroup of rotations about axis $\mathcal{A}$;

2. $\mathcal{P}(\mathbf{e})$, the subgroup of translations along the direction $\mathbf{e}$;

3. $\mathcal{H}(\mathcal{A}, p)$, the subgroup of rotations through an angle $\phi$ about axis $\mathcal{A}$ and translations $u$ along the direction of the same axis, translations and rotations being related by the pitch $p$ in the form $u=p \phi$;

4. $\mathcal{C}(\mathcal{A})$, the subgroup of independent rotations about and translations in the direction of axis $\mathcal{A}$;

5. $\mathcal{F}(\mathbf{u}, \mathbf{v})$, the subgroup of two independent translations in the directions of the distinct unit vectors $\mathbf{u}$ and $\mathbf{v}$, and one rotation about an axis normal to both $\mathbf{u}$ and $\mathbf{v}$;

6. $\mathcal{S}(O)$, the subgroup of rotations about point $O$.

7. $\mathcal{I}$, the identity subgroup;

8. $\mathcal{T}_{2}(\mathbf{u}, \mathbf{v})$, the planar-translation subgroup of translations in the directions of the two distinct unit vectors $\mathbf{u}$ and $\mathbf{v}$;

9. $\mathcal{T}_{3}$, the translation subgroup of translations in $3 \mathrm{D}$ space;

10. $\mathcal{Y}(\mathbf{e}, p)$, the subgroup of motions allowed by a screw of pitch $p$ and axis parallel to e undergoing arbitrary translations in a direction normal to e;

\footnotetext{
${ }^{1}$ An axis being a line, $\mathcal{A}$ is fully determined by either a point and a direction or two points.
} 
11. $\mathcal{X}(\mathbf{u})=\mathcal{F}(\mathbf{v}, \mathbf{w}) \bullet \mathcal{P}(\mathbf{u})$, the subgroup resulting of the product of the planar subgroup of plane normal to $\mathbf{u}$, i.e., $\mathbf{v} \cdot \mathbf{u}=\mathbf{w} \cdot \mathbf{u}=0$, and the prismatic subgroup of direction $\mathbf{u}$. Each element of this subgroup is thus characterized by the two translations $t_{v}, t_{w}$ and the angle $\phi$ of the planar subgroup plus the translation $t_{u}$ in the direction of $\mathbf{u}$. This subgroup is known as the Schönflies subgroup;

12. $\mathcal{D}$, the set of rigid-body displacements, each of whose elements is characterized by three independent translations and three independent rotations.

A kinematic bond is defined as a set of displacements stemming from the product of displacement subgroups, [5, 15]. Notice that a bond itself need not be a subgroup. For example, the universal bond

$$
\mathcal{U}(O)=\mathcal{R}\left(\mathcal{A}_{1}\right) \bullet \mathcal{R}\left(\mathcal{A}_{2}\right)
$$

where $\mathcal{A}_{1}$ and $\mathcal{A}_{2}$ are two axes intersecting at point $O$ at right angles, is not a subgroup. The name of this bond derives from its mechanical realization, namely, the universal joint. We denote a kinematic bond by $\mathcal{L}(i, n)$, where $i$ and $n$ stand for the integer numbers associated with the two end links of the bond.

A kinematic bond is realized by a kinematic chain, which is the result of the coupling of rigid bodies, called links, via kinematic pairs. When the coupling takes place in such a way that the two links share a common surface, a lower kinematic pair results; when the coupling takes place along a common line or a common point, a higher kinematic pair arises. Examples of higher kinematic pairs include gears and cams. П-joints are a special kind, not belonging to either lower- nor higher-kinematic pairs. We shall denote the subgroup associated with the lower kinematic pair coupling links $i$ and $i+1$ as $\mathcal{L}(i, i+1)$, a kinematic bond $\mathcal{L}(i, n)$ being obtained from the product of $n-i$ such subgroups, i.e.,

$$
\mathcal{L}(i, n)=\mathcal{L}(i, i+1) \bullet \mathcal{L}(i+1, i+2) \bullet \cdots \bullet \mathcal{L}(n-1, n)
$$

There are six basic lower kinematic pairs, namely (1) revolute $(R),(2)$ prismatic $(P),(3)$ helical $(\mathrm{H}),(4)$ cylindrical $(\mathrm{C}),(5)$ planar $(\mathrm{F})$, and (6) spherical $(\mathrm{S})$. These pairs are the generators of the displacement subgroups $\mathcal{R}(\mathcal{A}), \mathcal{P}(\mathbf{e}), \mathcal{H}(\mathcal{A}, p), \mathcal{C}(\mathcal{A}), \mathcal{F}(\mathbf{u}, \mathbf{v})$ and $\mathcal{S}(O)$, respectively. Although the foregoing displacement subgroups can be realized by their corresponding lower kinematic pairs, it is often possible to realize the displacement subgroups by appropriate kinematic chains. A common example is that of $\mathcal{C}(\mathcal{A})$ which, besides the $\mathrm{C}$ pair, can be realized by a suitable concatenation of a $\mathrm{P}$ and a $\mathrm{R}$ pair $[16$.

In addition to the first three lower kinematic pairs, we consider the П-joint [11, 12, 17], a parallelogram four-bar linkage, when synthesizing a SMG. 


\section{Type Synthesis of a Serial SMG}

In this section we introduce a novel method to identify the motions generated by a kinematic bond based on the displacement subgroups. This method is then used to produce a list of Schönflies-Motion Generators. As we focus on serial SMGs, let us introduce three premises:

Premise 1 Only manipulators composed of a combination of $\mathrm{R}, \mathrm{P}, \mathrm{H}$ and $\Pi$ joints are considered.

Premise 2 All $\mathrm{R}$ and $\mathrm{H}$ joints composing a serial SMG must have their axes parallel.

Premise 3 The direction of a $\mathrm{P}$ joint $\mathrm{E}$ need not be normal to the other joint axes composing a serial $S M G$.

The combination of subgroups, in general, can take place via the group product operation. Now we introduce

Definition 1 A kinematic chain composed of kinematic pairs $\mathrm{R}, \mathrm{P}, \mathrm{H}$ of axes parallel to vector $\mathbf{u}$ is said to be a class- $\mathcal{X}_{\mathbf{u}}$ chain.

For brevity, in the balance of the paper we denote by $R_{\mathbf{u}}$ any revolute pair of axis parallel to a unit vector $\mathbf{u}$, the same notation applying to $\mathrm{H}_{\mathbf{u}}$. Furthermore, two $\mathrm{H}_{\mathbf{u}}$ pairs of a class- $\mathcal{X}_{\mathbf{u}}$ chain are assumed to have distinct pitches, unless otherwise stated. $P_{\mathbf{u}}$ denotes, in turn, a prismatic pair with direction parallel to the unit vector $\mathbf{u}$.

Definition 2 The dimension of a group is the number of independent variables that uniquely describe one member of the group. When a kinematic bond is a group, its dimension is the degree of freedom of the underlying kinematic chain.

Moreover, the concept of dimension is readily extended to a kinematic bond, namely,

Definition 3 The dimension of a kinematic bond is the number of independent variables that uniquely describe one member of the bond. The dimension of a kinematic bond is the degree of freedom of the kinematic chain that realizes the bond.

For example, for the bond $\mathcal{U}$ introduced in eq.(1),

$$
\operatorname{dim}(\mathcal{U})=2
$$

\footnotetext{
${ }^{2} \mathrm{P}$ joints do not have an axis, but only a direction. For a terser discussion, we refer sometimes to the "axis" of a $\mathrm{P}$ joint, the implication being that a direction is meant.
} 
Assuming two kinematic bonds $\mathcal{L}_{1}$ and $\mathcal{L}_{2}$, it is noteworthy that:

$$
\begin{aligned}
\operatorname{dim}\left\{\mathcal{L}_{1} \bullet \mathcal{L}_{2}\right\} & \leq \operatorname{dim}\left\{\mathcal{L}_{1}\right\}+\operatorname{dim}\left\{\mathcal{L}_{2}\right\} \\
\operatorname{dim}\left\{\mathcal{L}_{1} \cap \mathcal{L}_{2}\right\} & \leq \min \left\{\operatorname{dim}\left\{\mathcal{L}_{1}\right\}, \operatorname{dim}\left\{\mathcal{L}_{2}\right\}\right\}
\end{aligned}
$$

As an illustration of inequality (3), let us define $\mathcal{L}_{i}$ as $\mathcal{S}\left(O_{i}\right)$, for $i=1,2, O_{i}$ denoting one point $O_{i}$. One has

$$
\operatorname{dim}\left[\mathcal{S}\left(O_{i}\right)\right]=3, \quad i=1,2
$$

and

$$
\operatorname{dim}\left[\mathcal{S}\left(O_{1}\right) \bullet \mathcal{S}\left(O_{2}\right)\right]=5<\operatorname{dim}\left[\mathcal{S}\left(O_{1}\right)\right]+\operatorname{dim}\left[\mathcal{S}\left(O_{2}\right)\right]=6
$$

That is, the dof of a serial array of two spherical joints is five, as the kinematic bond thus resulting — not a subgroup - is short of one dof to allow its end link a full six-dof motion: the end link is prevented from translating in the direction of the segment $\overline{O_{1} O_{2}}$.

To illustrate inequality (4), let us define $\mathcal{L}_{1}$ as $\mathcal{R}\left(\mathcal{A}_{1}\right)$ and $\mathcal{L}_{2}$ as $\mathcal{C}\left(\mathcal{A}_{2}\right)$, with $\mathcal{A}_{1}$ and $\mathcal{A}_{2}$ defined, in turn, as two parallel lines. Thus,

$$
\operatorname{dim}\left[\mathcal{R}\left(\mathcal{A}_{1}\right)\right]=1, \quad \operatorname{dim}\left[\mathcal{C}\left(\mathcal{A}_{2}\right)\right]=2, \quad \operatorname{dim}\left[\mathcal{R}\left(\mathcal{A}_{1}\right) \cap \mathcal{C}\left(\mathcal{A}_{2}\right)\right]=0
$$

Indeed, the parallel array of one $\mathrm{R}$ and one $\mathrm{C}$ joints, of parallel axes constrains completely the two coupled links, the kinematic chain of interest then degenerating into one isostatic structure.

It is noteworthy that, as a consequence of inequality (田), the degree of freedom of a Parallel Kinematics Machine (PKM) with $l$ limbs is, at most, equal to the dof of its limb with the lowest mobility.

\section{Rules for the Type-Synthesis of Serial SMGs}

This section aims at defining a set of rules for the type-synthesis of serial SMGs composed of only $\mathrm{R}, \mathrm{P}, \mathrm{H}$ and $\Pi$-joints. Let $\mathcal{X}(\mathbf{u})$ be the required Schönflies motion, i.e., the Schönflies subgroup about an axis parallel to the unit vector $\mathbf{u}$. In this vein, we provide below a set of rules in the form of lemmas and corollaries.

Lemma 1 The bond produced by any lower kinematic pair is idempotent, i.e., if $\mathcal{L}(\cdot)$ represents any of these bonds, then

$$
\mathcal{L}(\cdot) \bullet \mathcal{L}(\cdot)=\mathcal{L}(\cdot)
$$

Consequently,

Corollary 1 All twelve subgroups of $\mathcal{D}$ are idempotent. 
In particular, as far as Schönflies motion generation is concerned,

$$
\mathcal{R}(\mathcal{A}) \bullet \mathcal{R}(\mathcal{A})=\mathcal{R}(\mathcal{A}), \mathcal{P}(\mathbf{u}) \bullet \mathcal{P}(\mathbf{u})=\mathcal{P}(\mathbf{u}), \mathcal{H}(\mathcal{A}, p) \bullet \mathcal{H}(\mathcal{A}, p)=\mathcal{H}(\mathcal{A}, p)
$$

$\mathcal{A}$ being the axis of the corresponding pair, $\mathbf{u}$ its direction and $p$ the pitch of the $\mathrm{H}$ pair.

However, the product of two identical $\Pi$-bonds ${ }^{3}$ (serial array) is $\mathcal{T}_{2}$, i.e.,

$$
\Pi_{\mathbf{n}} \bullet \Pi_{\mathbf{n}}=\mathcal{T}_{2}(\mathbf{n})
$$

$\mathbf{n}$ being the axis normal to the plane of the two $\Pi$ joints generating the two identical $\Pi_{\mathbf{n}}$ bonds. From Corollary 1, it is apparent that:

Corollary 2 If a kinematic bond is a subgroup, then it is idempotent.

A kinematic bond that is not a subgroup is not necessarily idempotent.

Lemma 2 If a kinematic bond contains two prismatic pairs, of non-parallel directions, introducing a third prismatic pair of direction contained in the plane spanned by the direction vectors of the first two pairs does not affect the mobility of the original bond, e.g.

$$
\mathcal{P}(\mathbf{u}) \bullet \mathcal{P}(\mathbf{v}) \bullet \mathcal{P}(\mathbf{x})=\mathcal{P}(\mathbf{u}) \bullet \mathcal{P}(\mathbf{v})=\mathcal{T}_{2}(\mathbf{u}, \mathbf{v}), \quad \mathbf{x} \cdot(\mathbf{u} \times \mathbf{v})=0
$$

Lemma 3 The product of the bonds (subgroups, in this case) generated by coaxial revolute and cylindrical joints results in the same cylindrical bond:

$$
\mathcal{R}(\mathcal{A}) \bullet \mathcal{C}(\mathcal{A})=\mathcal{C}(\mathcal{A})
$$

Lemma 4 All revolute, cylindrical and helical joints of a serial chain must have their axes parallel in order to produce the Schönflies motion group $\mathcal{X}(\mathbf{u})$.

While, in general, the group product is not commutative,

Lemma 5 The product of any pair of bonds generated by a class- $\mathcal{X}_{\mathbf{u}}$ chain is commutative.

As a consequence of Lemma 5,

Corollary 3 The product of $n$ kinematic bonds generated by $n$ class- $\mathcal{X}_{\mathbf{u}}$ chains is immutable under a bond permutation.

\footnotetext{
${ }^{3}$ The bond of the $\Pi$-joint is denoted by a subscripted italic $\Pi$, the subscript denoting the unit vector normal to the plane of the joint.
} 


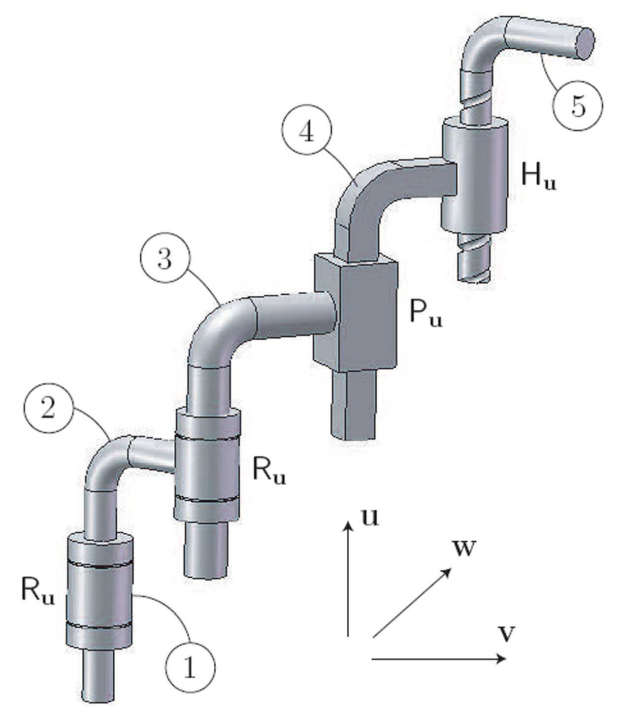

Figure 1: $\mathrm{R}_{\mathbf{u}} \mathrm{R}_{\mathbf{u}} \mathrm{P}_{\mathbf{u}} \mathrm{H}_{\mathbf{u}}$

Corrolary 3 implies that all chains obtained by a reordering of the joints generating $\mathcal{X}(\mathbf{u})$ are equivalent in terms of bond-generation. For example, let us assume five links, numbered from 1 to 5 , and coupled by four kinematic pairs, as shown in Fig. 1, all of the $\mathcal{X}_{\mathbf{u}}$-class. Due to the commutativity of the product operation within a displacement subgroup, then, any permutation of the joints of the kinematic chain of Fig. 目 is a SMG, generating the same $\mathcal{X}(\mathbf{u})$ subgroup. Now we have

Lemma 6 The minimum number of joints of the $\mathcal{X}_{\mathbf{u}}$-class or of the $\Pi_{\mathbf{n}}$ type, with $\mathbf{n}$ normal to $\mathbf{u}$, composing a $S M G$ is four.

Let us introduce one more definition:

Definition 4 Given two mutually orthogonal unit vectors $\mathbf{u}$ and $\mathbf{n}$, the set $\mathcal{S}_{\mathbf{u}}$ is defined as

$$
\mathcal{S}_{\mathbf{u}}=\left\{\mathrm{R}_{\mathbf{u}}, \mathrm{P}_{\mathbf{u}}, \mathrm{H}_{\mathbf{u}}, \Pi_{\mathbf{n}}\right\}
$$

That is, $\mathcal{S}_{\mathbf{u}}$ is nothing but $\mathcal{X}_{\mathbf{u}}$ augmented by the joint $\Pi_{\mathbf{n}}$.

Henceforth we focus on SMGs composed of four joints. Moreover, the four joints are either members of $\mathcal{S}_{\mathbf{u}}$ or of the $\mathrm{P}_{\mathbf{u}^{\perp}}$-type, where $\mathbf{u}^{\perp}$ is a unit vector normal to $\mathbf{u}$. Notice that the latter need not be identical to the unit vector $\mathbf{n}$ defining the $\Pi$ joint of $\mathcal{S}_{\mathbf{u}}$. Furthermore, if any of the joints of $\mathrm{R}_{\mathbf{u}^{-}}$or of $\mathrm{H}_{\mathbf{u}^{-}}$-type is repeated, then no two of the repeated joints are coaxial.

Lemma 7 If $\{\mathbf{u}, \mathbf{v}, \mathbf{w}\}$ denotes an orthonormal triad, then using $\mathrm{P}_{\mathbf{v}}$ or $\mathrm{P}_{\mathbf{w}}$ to obtain the Schönflies motion subgroup $\mathcal{X}(\mathbf{u})$ is equivalent, i.e., the chains composed of $\mathrm{P}_{\mathbf{v}}$ joints are equivalent to the ones composed of $\mathrm{P}_{\mathrm{w}}$ joints.

For example, 
- $\mathrm{R}_{\mathbf{u}} R_{\mathbf{u}} P_{\mathbf{v}} \mathrm{P}_{\mathbf{u}}$ and $\mathrm{R}_{\mathbf{u}} R_{\mathbf{u}} \mathrm{P}_{\mathbf{w}} \mathrm{P}_{\mathbf{u}}$ generate the same bond;

- $\mathrm{R}_{\mathbf{u}} \mathrm{R}_{\mathbf{u}} \mathrm{P}_{\mathbf{u}} \mathrm{P}_{\mathbf{v}}$ and $\mathrm{R}_{\mathbf{u}} \mathrm{R}_{\mathbf{u}} \mathrm{P}_{\mathbf{u}} \mathrm{P}_{\mathbf{w}}$ generate the same bond;

- $R_{\mathbf{u}} P_{\mathbf{v}} R_{\mathbf{u}} P_{\mathbf{u}}$ and $R_{\mathbf{u}} P_{\mathbf{w}} R_{\mathbf{u}} P_{\mathbf{u}}$ generate the same bond.

In the subsections below we list all independent kinematic chains producing $\mathcal{X}(\mathbf{u})$ with two, three and four joints of the set $\mathcal{S}_{\mathbf{u}}$.

\subsection{Serial SMGs with R and P Pairs}

A minimum list of SMGs producing $\mathcal{X}(\mathbf{u})$ and composed of only revolute and prismatic pairs obtained by using the foregoing rules is displayed in Table 1 .

\begin{tabular}{l|llll}
\hline \hline & \multicolumn{4}{|c}{ Manipulator } \\
\hline 1 & $\mathrm{R}_{\mathbf{u}}$ & $\mathrm{R}_{\mathbf{u}}$ & $\mathrm{R}_{\mathbf{u}}$ & $\mathrm{P}_{\mathbf{u}}$ \\
2 & $\mathrm{R}_{\mathbf{u}}$ & $\mathrm{R}_{\mathbf{u}}$ & $\mathrm{P}_{\mathbf{u}}$ & $\mathrm{P}_{\mathbf{v}}$ \\
3 & $\mathrm{R}_{\mathbf{u}}$ & $\mathrm{P}_{\mathbf{u}}$ & $\mathrm{P}_{\mathbf{v}}$ & $\mathrm{P}_{\mathbf{w}}$ \\
\hline \hline
\end{tabular}

Table 1: Minimum list of $\mathcal{X}_{\mathbf{u}}$-generators with $\mathrm{R}$ and $\mathrm{P}$ pairs

\subsection{Serial SMGs with R, P and H Pairs}

As shown by Lee and Hervé 四, the Schönflies subgroup can be generated by means of sequences of kinematic pairs and a generic expression of the decomposition of the Schönflies subgroup into a product of four one-dimensional subgroups, which are associated with the single-dof lower kinematic pairs, e.g.,

$$
\mathcal{X}(\mathbf{u})=\mathcal{H}\left(\mathcal{A}_{1}, p_{1}\right) \bullet \mathcal{H}\left(\mathcal{A}_{2}, p_{2}\right) \bullet \mathcal{H}\left(\mathcal{A}_{3}, p_{3}\right) \bullet \mathcal{H}\left(\mathcal{A}_{4}, p_{4}\right)
$$

with $\mathcal{A}_{i}$ passing through a point $A_{i}$ of position vector $\mathbf{a}_{i}$ and parallel to the unit vector $\mathbf{u}, p_{i}$ being the pitch of the $i$ th helical pair. In the foregoing expression, every $\mathcal{H}$ subgroup comprises both the $\mathcal{R}$ and $\mathcal{P}$ subgroups, the former when the pitch vanishes, the latter when the pitch becomes unbounded.

In order to obtain the minimum list of serial SMGs composed of any combination of R, $\mathrm{P}$ and $\mathrm{H}$ pairs, we have to follow the rules introduced in Subsection 4.1 along with the ones stated below: 
Lemma 8 The four pitches in Eq. (6) must be distinct. Indeed, if $p_{1}=p_{2}=p_{3}=p_{4}=p$, then

$$
\mathcal{H}\left(\mathcal{A}_{1}, p_{1}\right) \bullet \mathcal{H}\left(\mathcal{A}_{2}, p_{2}\right) \bullet \mathcal{H}\left(\mathcal{A}_{3}, p_{3}\right) \bullet \mathcal{H}\left(\mathcal{A}_{4}, p_{4}\right) \in \mathcal{Y}(\mathbf{u}, p)
$$

which is the 10th subgroup listed in Section 园. Each element of this subgroup is thus characterized by the two independent translations $t_{\mathbf{v}}, t_{\mathbf{w}}$ of directions perpendicular to $\mathbf{u}$, and either the rotation $\phi$ about this axis or the translation $t_{\mathbf{u}}$ in the direction of the axis.

Lemma 9 If a Schönflies-motion-generating bond is composed of two $\mathrm{H}$ and two $\mathrm{P}$ pairs, and the former have identical pitches, with axes parallel to $\mathbf{u}$, then none of the directions of the latter must be perpendicular to $\mathbf{u}$.

For a proof of this Lemma, the reader is referred to [4].

\begin{tabular}{|c|c|c|c|c|c|c|c|c|c|}
\hline $\mathrm{N}^{\mathrm{O}}$ & \multicolumn{4}{|c|}{ Manipulator } & $\mathrm{N}^{\mathrm{O}}$ & \multicolumn{4}{|c|}{ Manipulator } \\
\hline 1 & $\mathrm{R}_{\mathrm{u}}$ & $\mathrm{R}_{\mathrm{u}}$ & $\mathrm{R}_{\mathrm{u}}$ & & 2 & $\mathrm{R}_{\mathbf{u}}$ & $\mathrm{R}_{\mathrm{u}}$ & $\mathrm{R}_{\mathbf{u}}$ & $\mathrm{H}_{\mathrm{u}}$ \\
\hline 3 & $\mathrm{R}_{\mathbf{u}}$ & $\mathrm{R}_{\mathbf{u}}$ & $P_{u}$ & $P_{\mathbf{v}}$ & 4 & $\mathrm{R}_{\mathrm{u}}$ & $\mathrm{R}_{\mathrm{u}}$ & $\mathrm{P}_{\mathbf{u}}$ & $\mathrm{H}_{\mathbf{u}}$ \\
\hline 5 & $\mathrm{R}_{\mathbf{u}}$ & $\mathrm{R}_{\mathbf{u}}$ & $P_{\mathbf{v}}$ & $\mathrm{H}_{\mathrm{u}}$ & 6 & $\mathrm{R}_{\mathbf{u}}$ & $\mathrm{R}_{\mathrm{u}}$ & $\mathrm{H}_{\mathbf{u}}$ & $\mathrm{H}_{\mathbf{u}}$ \\
\hline 7 & $\mathrm{R}_{\mathrm{u}}$ & $\mathrm{P}_{\mathrm{u}}$ & $P_{v}$ & $P_{w}$ & 8 & $\mathrm{R}_{\mathbf{u}}$ & $P_{u}$ & $\mathrm{P}_{\mathrm{v}}$ & $\mathrm{H}_{\mathbf{u}}$ \\
\hline 9 & $\mathrm{R}_{\mathrm{u}}$ & $P_{u}$ & $\mathrm{H}_{\mathrm{u}}$ & $\mathrm{H}_{\mathbf{u}}$ & 10 & $\mathrm{R}_{\mathrm{u}}$ & $P_{v}$ & $P_{w}$ & $\mathrm{H}_{\mathbf{u}}$ \\
\hline 11 & $\mathrm{R}_{\mathrm{u}}$ & $\mathrm{P}_{\mathrm{v}}$ & $\mathrm{H}_{\mathrm{u}}$ & $\mathrm{H}_{\mathrm{u}}$ & 12 & $\mathrm{R}_{\mathrm{u}}$ & $\mathrm{H}_{\mathbf{u}}$ & $\mathrm{H}_{\mathrm{u}}$ & $\mathrm{H}_{\mathbf{u}}$ \\
\hline 13 & $P_{\mathbf{u}}$ & $\mathrm{P}_{\mathrm{v}}$ & $\mathrm{P}_{\mathrm{w}}$ & $\mathrm{H}_{\mathrm{u}}$ & 14 & $P_{\mathbf{u}}$ & $P_{v}$ & $\mathrm{H}_{\mathbf{u}}$ & $\mathrm{H}_{\mathrm{u}}$ \\
\hline 15 & $P_{u}$ & $\mathrm{H}_{\mathbf{u}}$ & $\mathrm{H}_{\mathrm{u}}$ & $\mathrm{H}_{\mathbf{u}}$ & 16 & $P_{v}$ & $P_{w}$ & $\mathrm{H}_{\mathrm{u}}$ & $\mathrm{H}_{\mathbf{u}}$ \\
\hline 17 & $P_{v}$ & $\mathrm{H}_{\mathrm{u}}$ & $\mathrm{H}_{\mathrm{u}}$ & $\mathrm{H}_{\mathrm{u}}$ & 18 & $\mathrm{H}_{\mathrm{u}}$ & $\mathrm{H}_{\mathbf{u}}$ & $\mathrm{H}_{\mathbf{u}}$ & $\mathrm{H}_{\mathrm{u}}$ \\
\hline
\end{tabular}

Table 2: Minimum list of $\mathcal{X}_{\mathbf{u}}$-generators with $\mathrm{R}, \mathrm{P}$, and $\mathrm{H}$ pairs

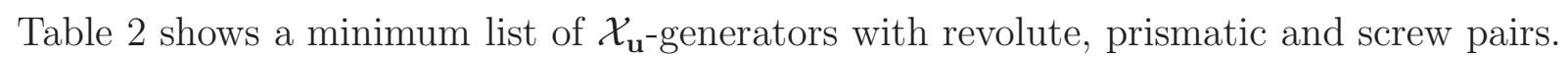

\subsection{Serial SMGs with R, P, H and $\Pi$ Joints}

To come up with a minimum list of SMGs with $\mathrm{R}, \mathrm{P}, \mathrm{H}$ and $\Pi$ joints, generating $\mathcal{X}(\mathbf{u})$, we have to follow the rules introduced in Subsections 4.1 and 4.2 plus the one below:

Lemma 10 The $S M G$ cannot contain more than two $\Pi_{\mathbf{u}}, \Pi_{\mathbf{v}}$ or $\Pi_{\mathbf{w}}$ pairs.

Table 3 shows a minimum list of serial SMGs, in order of ascending complexity, composed of any combination of $\mathrm{R}, \mathrm{P}, \mathrm{H}$ and $\Pi$ joints

\footnotetext{
${ }^{4}$ The reader is referred to http://www.irccyn.ec-nantes.fr/ caro/Publications/MMT/Figures3to7.pdf for a rendering of each of the 79 chains of Table 3 .
} 


\begin{tabular}{|c|c|c|c|c|c|c|c|c|c|}
\hline $\mathrm{N}^{\mathrm{O}}$ & \multicolumn{4}{|c|}{ Manipulator } & $K_{N}$ & $K_{L}$ & $K_{J}$ & $K_{B}$ & $K$ \\
\hline 1 & $\mathrm{R}_{\mathrm{u}}$ & $\mathrm{R}_{\mathrm{u}}$ & $\mathrm{R}_{\mathrm{u}}$ & $\mathrm{H}_{\mathbf{u}}$ & 0.5076 & 0 & 0.5941 & 0 & 0.2754 \\
\hline 2 & $\mathrm{R}_{\mathrm{u}}$ & $\mathrm{R}_{\mathbf{u}}$ & $\mathrm{R}_{\mathrm{u}}$ & $P_{u}$ & 0.5076 & 0 & 0.6425 & 0 & 0.2875 \\
\hline 3 & $\mathrm{R}_{\mathrm{u}}$ & $\mathrm{R}_{\mathbf{u}}$ & $\mathrm{H}_{\mathbf{u}}$ & $\mathrm{H}_{\mathbf{u}}$ & .5076 & 0 & 0.6649 & 0 & 0.2931 \\
\hline 4 & $\mathrm{R}_{\mathrm{u}}$ & $\mathrm{R}_{\mathbf{u}}$ & $P_{u}$ & $\mathrm{H}_{\mathrm{u}}$ & 0.5076 & 0 & 0.7133 & 0 & 0.3052 \\
\hline 5 & $\mathrm{R}_{\mathrm{u}}$ & $\mathrm{H}_{\mathrm{u}}$ & $\mathrm{H}_{\mathbf{u}}$ & $\mathrm{H}_{\mathbf{u}}$ & 0.5076 & 0 & 0.7357 & 0 & 0.3108 \\
\hline 6 & $\mathrm{R}_{\mathrm{u}}$ & $P_{u}$ & $\mathrm{H}_{\mathbf{u}}$ & $\mathrm{H}_{\mathrm{u}}$ & 0.5076 & 0 & 0.7841 & 0 & 0.3229 \\
\hline 7 & $\mathrm{H}_{\mathbf{u}}$ & $\mathrm{H}_{\mathrm{u}}$ & $\mathrm{H}_{\mathbf{u}}$ & $\mathrm{H}_{\mathrm{u}}$ & 0.5076 & 0 & 08064 & 0 & 0.3285 \\
\hline 8 & $P_{u}$ & $\mathrm{H}_{\mathrm{u}}$ & $\mathrm{H}_{\mathrm{u}}$ & $\mathrm{H}_{\mathrm{u}}$ & 0.5076 & 0 & 0.8548 & 0 & 0.3406 \\
\hline 9 & $\mathrm{R}_{\mathrm{u}}$ & $\mathrm{R}_{\mathrm{u}}$ & $P_{u}$ & $\mathrm{P}_{\mathrm{v}}$ & 0.5076 & 0 & 0.7617 & 0.2091 & 0.3696 \\
\hline 10 & $\mathrm{R}_{\mathrm{u}}$ & $\mathrm{R}_{\mathbf{u}}$ & $P_{v}$ & $\mathrm{H}_{\mathrm{u}}$ & 0.5076 & 0 & & & 0.3830 \\
\hline 11 & $P_{v}$ & $\mathrm{H}_{\mathbf{u}}$ & $\mathrm{H}_{\mathbf{u}}$ & $\mathrm{H}_{\mathrm{u}}$ & 0.5076 & 0 & 0.8548 & 0.2091 & 0.3929 \\
\hline 12 & $\mathrm{R}_{\mathrm{u}}$ & $P_{v}$ & $\mathrm{H}_{\mathrm{u}}$ & $\mathrm{H}_{\mathrm{u}}$ & 0.5076 & 0 & 0.7841 & 0.3109 & 0.4006 \\
\hline 13 & $\mathrm{R}_{\mathrm{u}}$ & $P_{u}$ & $P_{v}$ & $\mathrm{H}_{\mathrm{u}}$ & 0.5076 & 0 & 8325 & 0.3109 & 0.4127 \\
\hline 14 & $\mathrm{R}_{\mathrm{u}}$ & $P_{u}$ & $P_{v}$ & $P_{w}$ & 0.5076 & 0 & & & 0.4138 \\
\hline 15 & $\mathrm{R}_{\mathrm{u}}$ & $P_{v}$ & $P_{w}$ & $\mathrm{H}_{\mathbf{u}}$ & 0.5076 & 0 & & 0.3291 & 0.4173 \\
\hline 16 & $P_{v}$ & $P_{w}$ & $\mathrm{H}_{\mathrm{u}}$ & $\mathrm{H}_{\mathrm{u}}$ & 0.5076 & 0 & 0.9032 & 0.2668 & 0.4194 \\
\hline 17 & $P_{u}$ & $P_{v}$ & $\mathrm{H}_{\mathbf{u}}$ & $\mathrm{H}_{\mathbf{u}}$ & 0.5076 & 0 & .9032 & 0.3109 & 0.4304 \\
\hline 18 & $\mathrm{R}_{\mathrm{u}}$ & $\mathrm{R}_{\mathrm{u}}$ & $\mathrm{H}_{\mathrm{u}}$ & $\Pi_{\mathbf{u}}$ & & & & 0 & 0.4339 \\
\hline 19 & $\mathrm{R}_{\mathrm{u}}$ & $\mathrm{R}_{\mathrm{u}}$ & $P_{u}$ & $\Pi_{\mathbf{u}}$ & 0.7106 & & 0.5167 & 0 & 0.4408 \\
\hline 20 & $\mathrm{R}_{\mathrm{u}}$ & $\mathrm{H}_{\mathrm{u}}$ & $\mathrm{H}_{\mathrm{u}}$ & $\Pi_{\mathbf{u}}$ & 0.7106 & 0.5358 & 0.5295 & 0 & 0.4440 \\
\hline 21 & $P_{u}$ & $P_{v}$ & $P_{w}$ & $\mathrm{H}_{\mathrm{u}}$ & 0.5076 & 0 & 0.9516 & 0.3291 & 0.4471 \\
\hline 22 & $\mathrm{R}_{\mathrm{u}}$ & $P_{u}$ & $\mathrm{H}_{\mathrm{u}}$ & $\Pi_{\mathbf{u}}$ & 0.7106 & & & 0 & 0.4509 \\
\hline 23 & $\mathrm{H}_{\mathrm{u}}$ & $\mathrm{H}_{\mathrm{u}}$ & $\mathrm{H}_{\mathbf{u}}$ & $\Pi_{\mathbf{u}}$ & 0.7106 & & 0.5699 & 0 & 0.4541 \\
\hline 24 & $P_{u}$ & $\mathrm{H}_{\mathrm{u}}$ & $\mathrm{H}_{\mathrm{u}}$ & $\Pi_{\mathbf{u}}$ & 0.7106 & 0.5358 & 0.5976 & 0 & 0.4610 \\
\hline 25 & $\mathrm{R}_{\mathrm{u}}$ & $\mathrm{R}_{\mathrm{u}}$ & $\mathrm{R}_{\mathrm{u}}$ & $\Pi_{\mathbf{v}}$ & 0.7106 & & & & 0.4760 \\
\hline 26 & $\mathrm{R}_{\mathrm{u}}$ & $\mathrm{R}_{\mathrm{u}}$ & $\mathrm{H}_{\mathrm{u}}$ & $\Pi_{\mathbf{v}}$ & 0.7106 & 0.5358 & 0.4891 & 0.2091 & 0.4861 \\
\hline 27 & $\mathrm{R}_{\mathrm{u}}$ & $\mathrm{R}_{\mathrm{u}}$ & $P_{v}$ & $\Pi_{\mathbf{v}}$ & 0.71 & & & 0.1893 & 0.4881 \\
\hline 28 & $\mathrm{R}_{\mathrm{u}}$ & $\mathrm{R}_{\mathbf{u}}$ & $\mathrm{P}_{\mathrm{u}}$ & $\Pi_{\mathbf{v}}$ & 0.7106 & 0.5358 & 0.5167 & 0.2091 & 0.4931 \\
\hline 29 & $\mathrm{R}_{\mathrm{u}}$ & $\mathrm{H}_{\mathbf{u}}$ & $\mathrm{H}_{\mathrm{u}}$ & $\Pi_{\mathbf{v}}$ & 0.7106 & 0.5358 & 0.5295 & 0.2091 & 0.4962 \\
\hline 30 & $\mathrm{R}_{\mathrm{u}}$ & $P_{u}$ & $\mathrm{H}_{\mathrm{u}}$ & $\Pi_{\mathbf{v}}$ & 0.7106 & 0.5358 & 0.5571 & 0.2091 & 0.5032 \\
\hline 31 & $\mathrm{R}_{\mathrm{u}}$ & $P_{u}$ & $P_{v}$ & $\Pi_{\mathbf{v}}$ & & 0.5358 & 0.5848 & 0.1893 & 0.5051 \\
\hline 32 & $\mathrm{H}_{\mathrm{u}}$ & $\mathrm{H}_{\mathrm{u}}$ & $\mathrm{H}_{\mathrm{u}}$ & $\Pi_{\mathbf{v}}$ & 0.7106 & 0.5358 & 0.5699 & 0.2091 & 0.5064 \\
\hline 33 & $P_{u}$ & $\mathrm{H}_{\mathrm{u}}$ & $\mathrm{H}_{\mathrm{u}}$ & $\Pi_{\mathbf{v}}$ & 0.7106 & 0.5358 & 0.5976 & 0.2091 & 0.5133 \\
\hline 34 & $P_{v}$ & $\mathrm{H}_{\mathrm{u}}$ & $\mathrm{H}_{\mathrm{u}}$ & $\Pi_{\mathbf{u}}$ & 0.7106 & 0.5358 & 0.5976 & 0.2091 & 0.5133 \\
\hline
\end{tabular}




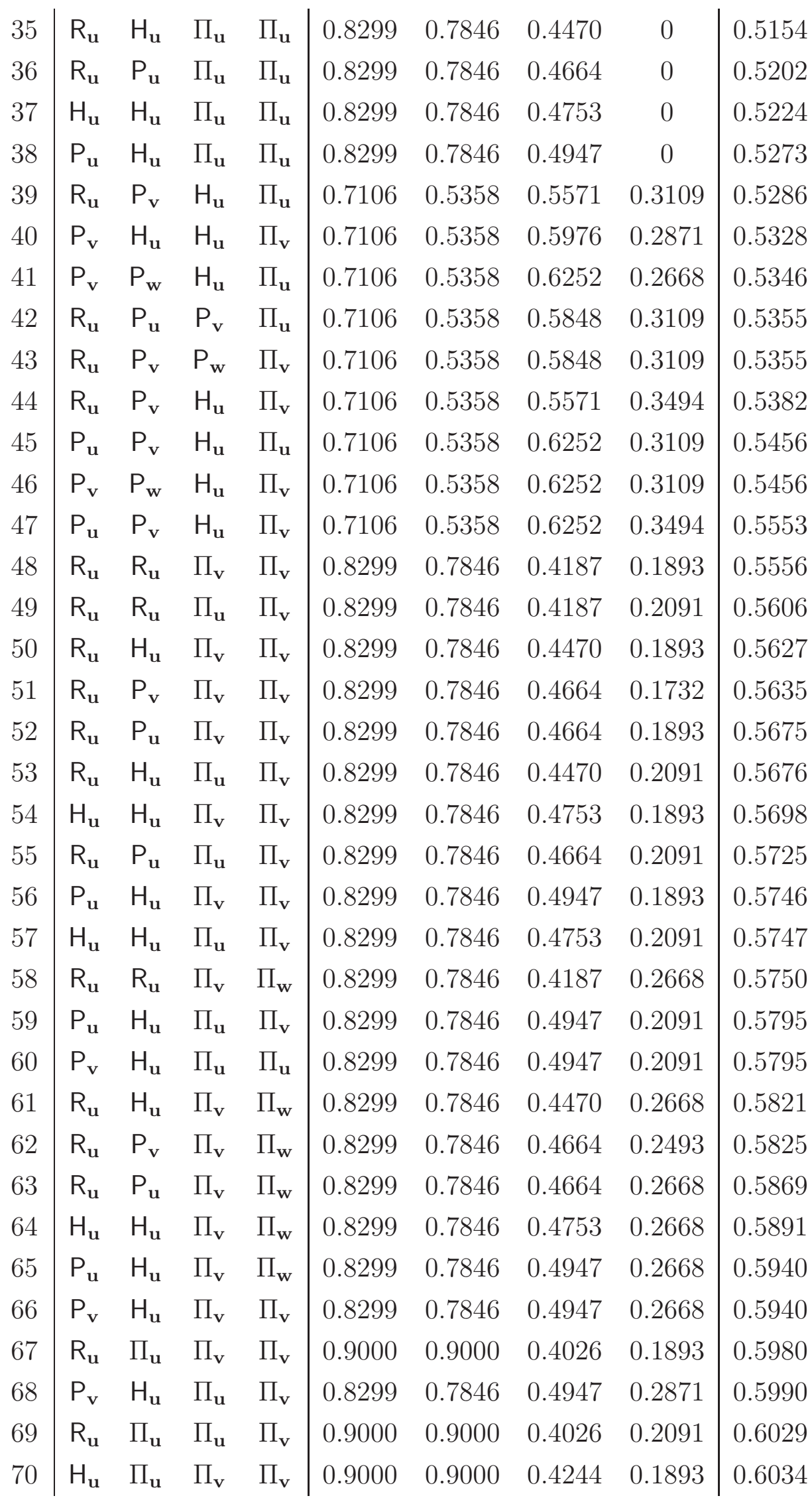




\begin{tabular}{l|llll|llll|l}
71 & $\mathrm{P}_{\mathbf{v}}$ & $\mathrm{H}_{\mathbf{u}}$ & $\Pi_{\mathbf{v}}$ & $\Pi_{\mathbf{w}}$ & 0.8299 & 0.7846 & 0.4947 & 0.3109 & 0.6050 \\
72 & $\mathrm{R}_{\mathbf{u}}$ & $\mathrm{P}_{\mathbf{v}}$ & $\Pi_{\mathbf{u}}$ & $\Pi_{\mathbf{v}}$ & 0.8299 & 0.7846 & 0.4664 & 0.3494 & 0.6076 \\
73 & $\mathrm{H}_{\mathbf{u}}$ & $\Pi_{\mathbf{u}}$ & $\Pi_{\mathbf{u}}$ & $\Pi_{\mathbf{v}}$ & 0.9000 & 0.9000 & 0.4244 & 0.2091 & 0.6084 \\
74 & $\mathrm{R}_{\mathbf{u}}$ & $\Pi_{\mathbf{v}}$ & $\Pi_{\mathbf{w}}$ & $\Pi_{\mathbf{w}}$ & 0.9000 & 0.9000 & 0.4026 & 0.2341 & 0.6092 \\
75 & $\mathrm{R}_{\mathbf{u}}$ & $\Pi_{\mathbf{v}}$ & $\Pi_{\mathbf{v}}$ & $\Pi_{\mathbf{w}}$ & 0.9000 & 0.9000 & 0.4026 & 0.2493 & 0.6130 \\
76 & $\mathrm{H}_{\mathbf{u}}$ & $\Pi_{\mathbf{v}}$ & $\Pi_{\mathbf{w}}$ & $\Pi_{\mathbf{w}}$ & 0.9000 & 0.9000 & 0.4244 & 0.2341 & 0.6146 \\
77 & $\mathrm{R}_{\mathbf{u}}$ & $\Pi_{\mathbf{u}}$ & $\Pi_{\mathbf{v}}$ & $\Pi_{\mathbf{w}}$ & 0.9000 & 0.9000 & 0.4026 & 0.2668 & 0.6173 \\
78 & $\mathrm{H}_{\mathbf{u}}$ & $\Pi_{\mathbf{v}}$ & $\Pi_{\mathbf{v}}$ & $\Pi_{\mathbf{w}}$ & 0.9000 & 0.9000 & 0.4244 & 0.2493 & 0.6184 \\
79 & $\mathrm{H}_{\mathbf{u}}$ & $\Pi_{\mathbf{u}}$ & $\Pi_{\mathbf{v}}$ & $\Pi_{\mathbf{w}}$ & 0.9000 & 0.9000 & 0.4244 & 0.2668 & 0.6228 \\
\hline \hline
\end{tabular}

Table 3: Minimum list of $\mathcal{X}_{\mathbf{u}}$-generators with $\mathrm{R}, \mathrm{P}, \mathrm{H}$, and $\Pi$ joints, in order of ascending complexity

\section{Complexity Evaluation}

The 79 chains recorded in Table 3 are listed in order of ascending complexity, which is evaluated by means of a formulation provided in [16]. The complexity $K \in[0,1]$ of a kinematic chain is defined as a convex combination [18] of its various partial complexities, namely,

$$
K=w_{N} K_{N}+w_{L} K_{L}+w_{J} K_{J}+w_{B} K_{B}
$$

where $K_{N} \in[0,1]$ is the joint-number complexity, $K_{L} \in[0,1]$ the loop-complexity, $K_{J} \in[0,1]$ the joint-type complexity, and $K_{B} \in[0,1]$ the link diversity. A novel formulation of the latter is given in 20]. Furthermore, $w_{J}, w_{N}, w_{L}$, and $w_{B}$ denote their corresponding weights, such that $w_{J}+w_{N}+w_{L}+w_{B}=1$.

\section{$5.1 \quad$ Joint-Number Complexity $K_{N}$}

The joint-number complexity $K_{N}$ is defined as:

$$
K_{N}=1-\exp \left(-q_{N} N\right)
$$

where $N$ is the number of joints used in the chain at hand and $q_{N}$ is the resolution parameter, to be adjusted according to the resolution required, an issue that is discussed in Subsection 5.5 .

The joint-number complexity of the 79 chains is recorded in the third column of Table 3 . As a $\Pi$-joint is composed of four revolute joints, and the maximum number of $\Pi$-joints in the 
79 chains under study is equal to three, the maximum number of joints $N_{\max }$ per chain is equal to 13 , i.e., $N_{\max }=13$.

\subsection{Loop Complexity $K_{L}$}

The loop complexity $K_{L}$ of a robot is defined as:

$$
K_{L}=1-\exp \left(-q_{L} L\right) ; \quad L=l-l_{m}
$$

where $l$ is the number of kinematic loops in the robot, $l_{m}$ is the minimum number of loops required to produce a special displacement group or subgroup and $q_{L}$ is the corresponding resolution parameter. Within the scope of this paper $l_{m}=0$, as the Schönflies displacement subgroup can be produced by a single-open-chain. Moreover, the maximum number of loops in the 79 chains under study is equal to three, i.e., $L_{\max }=3$. The loop complexity of the 79 chains is recorded in the fourth column of Table 3 .

\section{$5.3 \quad$ Joint-Type Complexity $K_{J}$}

Joint-type complexity $K_{J}$ is that associated with the type of LKPs used in a kinematic chain. As the 79 chains are composed of revolute, prismatic, helical and $\Pi$-joints only, the $\Pi$-joints being composed of four revolute joints, we define this complexity as

$$
K_{J}=\frac{1}{n}\left[\left(n_{R}+4 n_{\Pi}\right) K_{G \mid R}+n_{P} K_{G \mid P}+n_{H} K_{G \mid H}\right]
$$

where $n_{R}, n_{P}, n_{H}$ and $n_{\Pi}$ are the numbers of revolute, prismatic, helical and $\Pi$-joints, respectively, while $n=n_{R}+n_{P}+n_{H}+4 n_{\Pi}$ and $K_{G \mid \mathrm{x}}$ the geometric complexity of the pair x, as introduced in 20]: $K_{G \mid R}=0.5234, K_{G \mid P}=1$ and $K_{G \mid H}=0.8064$. The joint-type complexity of the 79 chains is recorded in the fifth column of Table 3 .

\subsection{Link Diversity $K_{B}$}

At the conceptual design stage, partial information about the geometric relations between neighboring joints is available. The five possible link layouts of the two joint axes of a binary link described in Fig. 2 were reported in [20]. As the joint axes of the 79 chains are either parallel or orthogonal, we will distinguish only two types of link layouts in this paper, i.e., parallelism and perpendicularity. Besides, as $\Pi$-joints are composed of four revolute joints with parallel axes, it turns out that three constraints of parallelism have to be satisfied for their realization. Moreover, we borrow the concept of entropy from molecular thermodynamics and 


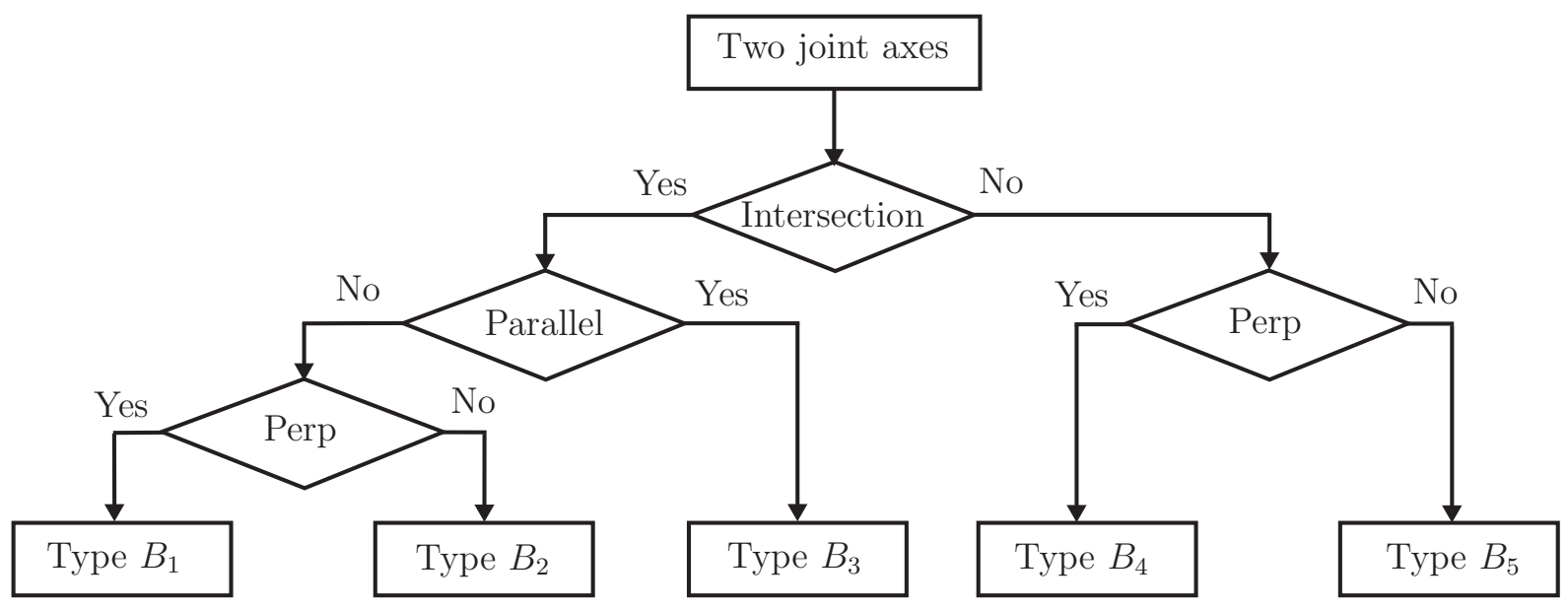

Figure 2: Binary tree displaying possible link topologies

from information theory [21] to evaluate the effect of geometric-constraint diversity, at the conceptual stage. In this vein, we define the geometric-constraint diversity as:

$$
K_{B}=\frac{B}{B_{\max }}, \quad B=-\sum_{i=1}^{c} b_{i} \log _{2}\left(b_{i}\right), \quad b_{i}=\frac{M_{i}}{\sum_{i=1}^{c} M_{i}}
$$

where $B$ is the entropy of the link layouts and $B_{\max }$ is the maximum possible value of $B$, with $B_{\max }=\log _{2}(5)=2.32$ bits [20], $c$ is the number of distinct joint-constraint types used in a concept and $M_{i}$ is the number of instances of each type of joint-constraints. The link diversity of the 79 chains is recorded in the sixth column of Table 3.

\subsection{Definition of the Resolution Parameters}

Two resolution parameters $q_{N}$ and $q_{L}$, introduced in Subsections 5.1 and 5.2, provide an appropriate resolution for the complexity at hand. Since the foregoing formulation is intended to compare the complexities of 79 kinematic chains, it is reasonable to assign a complexity of 0.9 to the chain with maximum complexity, and hence, evaluate the normalizing constant, i.e., for $J=N, L$,

$$
q_{J}=\left\{\begin{array}{lll}
-\ln (0.1) / J_{\max }, & \text { for } & J_{\max }>0 \\
0, & \text { for } & J_{\max }=0
\end{array}\right.
$$

Consequently, $q_{N}=0.1771$ and $q_{L}=0.7675$.

Finally, the total complexity $K$ of the 79 chains is recorded in the seventh column of Table 3 


\section{Conclusions}

The paper reported on the conceptual design of the architecture of serial Schönflies-Motion Generators. A set of synthesis rules, in the form of lemmas, was proposed to determine a minimum list of chains capable of producing Schönflies motions. The list is composed of 79 serial Schönflies-Motion Generators carrying any possible combination of R, P, H and $\Pi$ joints. Finally, a quantitative concept-evaluation framework, proposed in an earlier work, was applied to order the topologies obtained, from simplest to most complex.

It is noteworthy that the $\mathcal{X}_{\mathbf{u}}$ chains bearing $\Pi$ joints turn out to have the largest complexity, which may explain why only a handful of commercial SCARA systems of the serial type is equipped with this kind of joints. Robots of this type that bear $\Pi$ joints can be cited: OKURA Robotic Palletizer - Model A700, FANUC M-420iA, M-421iA and M-410iB robots, KUKA KR 40 PA, ABB IRB 260 and IRB 660 robots. These robots are intented to manipulate moderate to heavy loads.

We have included $\mathcal{X}_{\mathbf{u}}$ chains with $\Pi$ joints because, even if for serial SMGs they do not offer a great advantage, this type of chains occur frequently in parallel SMGs. The advantage that these chains offer lies in their stiffness and their ability to produce pure translations between the two links to which they are attached. As passive joints, parallelogram linkages in which the revolutes of planar four-bar linkages have been replaced by orientable pin joints, offer the advantage of having links that work under purely axial load.

\section{Acknowledgements}

This work was conducted with the support of the Idea to Innovation (I2I) Program of Canada's Natural Sciences and Engineering Research Council (NSERC). The partial support of the NSERC Design Engineering Chair at McGill University (2003-2008) and of the James McGill Professorship of Mechanical Engineering, held by the fourth author, are dutifully acknowledged.

\section{References}

[1] Makino, H., Kato, A. and Yamazaki, Y. (2007). "Research and Commercialization of SCARA Robot-The Case of Industry-University Joint Research and Development-," International Journal of Automation Technology, 1, pp. 61-67.

[2] Bottema, O. and Roth, B. (1979). Theoretical Kinematics, North Holland, Amsterdam. 
[3] Hervé, J. (1999). "The Lie Group of Rigid Body Displacements, a Fundamental Tool for Mechanical Design," Mech. Mach. Theory, 34, pp. 719-730.

[4] Lee, C-C. and Hervé, J.M. (2005). "On the Enumeration of Schoenflies Motion Generators," The Ninth IFToMM International Symposium on Theory of Machines and Mechanisms, Bucharest, Romania, September 1-4, SYROM 2005.

[5] Angeles, J. (2004). "The Qualitative Synthesis of Parallel Manipulators," ASME Journal of Mechanical Design, 126(4), pp. 617-624.

[6] Angeles, J., Caro, S., Khan, W.A. and Morozov, A. (2006). "The Design and Prototyping of an Innovative Schönflies Motion Generator," Proceedings of the IMechE Part C, Journal of Mechanical Engineering Science, special issue: Kinematics, Kinematic Geometry and their applications, 220(C7), July 2006, pp. 935-944.

[7] Lee, C-C. and Herve, J.M. (2006). "Translational Parallel Manipulators with Doubly Planar Limbs," Mech. Mach. Theory, 41(4), pp. 433-455.

[8] Lee, C-C. and Hervé, J.M. (2009). "Type Synthesis of Schoenflies Motion Generators," Mech. Mach. Theory, 44(10), pp. 1980-1997.

[9] Clavel, R. (1988). Delta, a Fast Robot with Parallel Geometry, Proc. 18th Int. Symp. Industrial Robots, Lausanne, pp. 91-100.

[10] Pierrot, F., Company, O. and Marquet, F. (2003). "H4: a High Speed 4 dof Parallel Robot." Synthesis, Modelling and Control Issues, IEEE Transactions on Robotics and Automation, 19(3), June 2003, pp. 411-420.

[11] Dietmaier, P. (1992). "Inverse Kinematics of Manipulators with 3 Revolute and 3 Parallelogram Joints," Proc. ASME 22nd Biennial Mechanisms Conference, Scottsdale, AZ, Sept. 13-16, 45, pp. 35-40.

[12] Hervé, J. M., and Sparacino, F. (1992). "Star, a New Concept in Robotics," Proc. 3rd Int. Workshop on Advances in Robot Kinematics, September 79, Ferrara, pp. 176-183.

[13] Wohlhart, K. (1992). "Displacement Analysis of the General Spatial Parallelogram Manipulator," Proc. 3rd International Workshop on Advances in Robot Kinematics, Ferrara, Italy, pp. 104-111.

[14] Macdonald, I.D. (1968). The Theory of Groups, Oxford University Press, London. 
[15] Hervé, J. (1978). "Analyse structurelle des mécanismes par groupes de déplacements," Mech. Mach. Theory, 13, pp. 437-450.

[16] Khan, W.A., Caro, S., Pasini, D. and Angeles, J. (2006a). "Complexity-Based Rules for the Conceptual Design of Robotic Architectures," $10^{\text {th }}$ International Symposium on Advances in Robot Kinematics, June 25-29, 2006, Ljubljana, Slovenia.

[17] Wohlhart, K., (1991). Der homogene Paralleltrieb-Mechanismus, Mathematica Pannonica, 2(2), pp. 59-76.

[18] Boyd, S. and Vandenberghe, L. (2004). Convex Optimization, Cambridge University Press, Cambridge.

[19] Khan, W.A., Caro, S., Pasini, D. and Angeles, J. (2006b). "Complexity Analysis of Curves and Surfaces: Application to the Geometry Complexity of Lower Kinematic Pairs," Department of Mechanical Engineering and Centre for Intelligent Machines, Technical Report, McGill University, Montreal.

[20] Khan, W.A., Caro, S., Angeles, J. and Pasini, D. (2007). "A Formulation of Complexity Based Rules for the Preliminary Design Stage of Robotic Architectures," The International Conference on Engineering Design, August 28-31, Paris, France.

[21] Shannon, C.E. and Weaver, W. (1998). The Mathematical Theory of Communication, (first published in 1949), University of Illinois Press, Urbana and Chicago. 